\title{
Proposal for a Photometric System for the Classification of Carbon Stars
}

\section{ULDIS DZERVITIS}

\section{Radioastrophysical Observatory, Riga, Latvia}

Because of the presence of molecular bands in the spectra of carbon stars resulting from their peculiar atmospheric compositions, most photometric systems are of limited value in determining the physical and chemical parameters of their atmospheres. Therefore, for the photometric classification of carbon stars in the visual and near infrared regions, a medium bandwidth (10-25 $\mathrm{nm}$ ) photometric system is proposed in which the locations of pass-bands are in conformity with the spectral peculiarities, and which is useful for small and moderate-sized telescopes and performable with interference filters and standard photomultipliers.

Integrating spectrophotometric records of about $60 \mathrm{~N}$ and late $\mathrm{R}$ stars with a systematic, step-by-step variation of the location and width of the proposed bands, a search was made for the highest resolution color-index diagrams. Five primary and two supplementary filters were selected, as follows:

Characteristics of the proposed system

\begin{tabular}{cll}
\hline Filter & Width $(\mathrm{nm})$ & Measured feature \\
\hline$\Phi_{51}$ & $505-520$ & C $_{2}$ Swan bands $\Delta v=0$ \\
$\Phi_{53}$ & $520-540$ & Quasicontinuum \\
$\Phi_{65}$ & $640-655$ & Quasicontinuum \\
$\Phi_{78}$ & $770-790$ & Quasicontinuum \\
$\Phi_{80}$ & $790-820$ & CN red system $\Delta v=2$ \\
\multicolumn{3}{c}{ Supplementary filters } \\
$\Phi_{59}$ & $585-595$ & Na D resonance doublet \\
$\Phi_{89}$ & $870-900$ & Quasicontinuum \\
\hline
\end{tabular}

The five primary bands of the system provide two color-indices representing effective temperature and two molecular indices $\left(\mathrm{C}_{2}\right.$ and $\left.\mathrm{CN}\right)$ which - as one can see from simplified equations of the thermochemical equilibrium in the very limited effective temperature interval of cool carbon stars $(2500-3000 \mathrm{~K})$ - can be treated as indicators of $\mathrm{C} / \mathrm{O}$ and $\mathrm{N} / \mathrm{H}$. The supplementary filters provide the possibility of measuring one more pseudocontinuum color-index and the $\mathrm{Na} \mathrm{D}$ resonance doublet intensity - a classical temperature indicator in carbon-star spectra. 